\title{
SWIFT PUBLICATION STATISTICS AND THE COMPARISON WITH OTHER MAJOR OBSERVATORIES
}

\author{
S. Savaglio ${ }^{1}$ and U. Grothkopf ${ }^{2}$
}

\begin{abstract}
The gamma-ray bursts (GRB) Swift satellite was launched at the end of 2004 and is funded until 2014. Its $\gamma$-ray, X-ray, and opticalUV instruments discover and localize about 100 GRBs per year. We report on the success of this mission by counting the number of papers with Swift data and their impact (i.e., number of citations to those papers) for the publication years 2005 to 2011. In the first year, the number of papers was 24 , and it steadily increased to 287 in the year 2011, reaching Keck. If this trend continues, before the end of the mission Swift may be approaching XMM-Newton and Chandra, with $\sim 400$ publications. Science topics of Swift publications have widened over time, and in 2011 almost 3/4 of all publications were about other energetic targets, such as AGN, novae, supernovae, X-ray binaries, pulsars, massive and stellar black holes.
\end{abstract}

\section{Introduction}

Bibliometrics is one quantitative approach to evaluate the success for individual scientists, research institutes, or universities. It counts the number of papers of these entities, and their impact through the number of citations to their papers.

Here we present the first complete bibliometric investigation for the years 2005-2011 of the $\gamma$-ray burst (GRB) mission Swift (Gehrels et al. 2004). Swift was launched at the end of 2004, while the first paper appeared in 2005. Its bibliometrics is compared with major observatories. For a full description of the method and details, see Savaglio \& Grothkopf (2012).

\section{Methodology}

For Swift, VLT, HST, Gemini and Subaru, papers are selected consistently. Only papers that use data are counted; theoretical papers are not included. Bibliometrics for XMM-Newton and Chandra is performed differently and their numbers are

\footnotetext{
1 MPI f. Extraterrestrial Physics, Garching bei München, Germany

2 European Southern Observatory, Garching bei München, Germany
} 


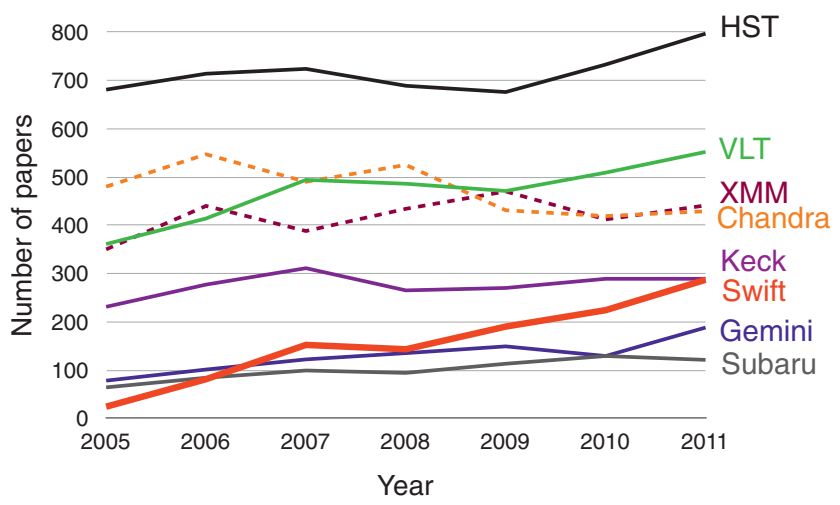

Fig. 1. Number of papers published based on data from different observatories, for the years 2005-2011. Swift data where first published in 2005 (thick red line), few months after its launch, in November 2004. Chandra and XMM-Newton are represented as dashed lines because here bibliometrics is measured using different criteria, which give a higher number of publications.

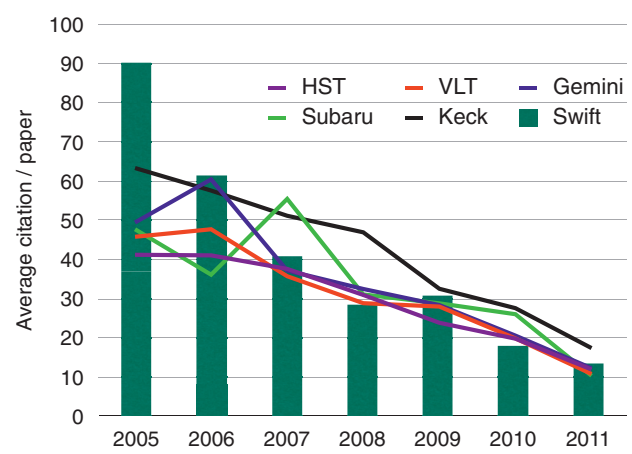

Fig. 2. Number of citations per paper for Swift (green histogram), HST, VLT, Gemini, Subaru and Keck, for the years 2005-2011.

generally higher than what we would get with our method. We used FUSE (FullText-Search tool), developed and maintained by the ESO Library (Erdmann \& Grothkopf 2010; Grothkopf \& Meakins 2012) to identify possible Swift papers. These are then carefully inspected to make sure that Swift data were used.

\section{Number of publications and citations}

The first Swift data papers were published in 2005, a few months after the launch (Fig. 1). With a steady and steep increase, the satellite produced 287 papers in 2011, many more than Gemini, twice those of Subaru, and reached Keck, one of the most successful observatories ever. After 7 years of operation, the plateau has 


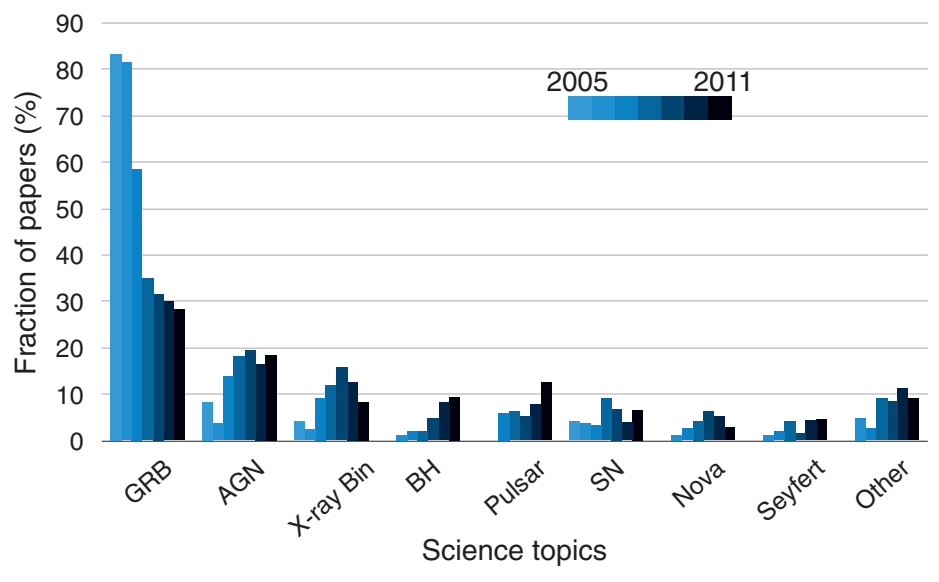

Fig. 3. Fraction of papers over the total for Swift ordered according to the science topic, for the years 2005-2011. An AGN paper is also dealing with distant QSOs, BL Lacs or blazers. $\mathrm{BH}$ indicates massive or stellar black holes. SN includes supernovae and supernova remnants.

not yet been reached; the total number of papers is 1101 . We obtained citations to data papers for all these observatories, for publication years 2005-2011 (Fig. 2). Swift papers are on average cited as often or more frequently than papers from other major observatories. In November 2012, the average number is 28.3 citations per paper. Among the top 150 most cited Swift publications, $26(\sim 17 \%)$ were published in Nature and Science, much more than what is generally obtained by other observatories $(5 \%-8 \%)$.

\section{Science topics}

While the main goal of Swift is to detect GRBs, the satellite also observes other energetic events (Fig. 3). In the first two years, over $80 \%$ of the publications were GRB papers, and almost $60 \%$ in 2007. During the past 4 yours, 2008-2011, the science done by Swift was mostly non-GRB (which droped to $28 \%-35 \%$ ). A large fraction is dedicated to galactic sources: $\sim 1 / 4$ of the papers in 2008-2011 are about X-ray binaries, pulsars, supernovae. Very popular are AGNs, with almost $20 \%$ of all publications.

\section{References}

Erdmann, C., \& Grothkopf, U., 2010, in: Library and Information Services in Astronomy VI: 21st Century Astronomy Librarianship, From New Ideas to Action, ASP Conf. Ser., 433, 81

Gehrels, N., Chincarini, G., Giommi, P., et al., 2004, ApJ, 611, 1005

Grothkopf, U., \& Meakins, S., 2012, The Messenger, 147, 41

Savaglio, S., \& Grothkopf, U., 2012, PASP, submitted 
